\title{
Aula invertida, TPACK y TAC: un camino hacia el aprendizaje
}

\section{Flipped Classroom, TPACK and TAC: A Path To Learning}

Recibido: 22 de septiembre de 2021 | Aprobado: 27 de noviembre de 2021

\section{Resumen}

La presente investigación se enmarca en la asignatura Innovación Educativa: cien estudiantes de las Licenciaturas en Matemáticas / Química, con orientación a la Educación. El problema se configura en torno a tres aspectos fundamentales: con frecuencia, incluso en la modalidad virtual, predomina el modelo educativo tradicional; la tecnología se usa como un recurso para la enseñanza, más que para el aprendizaje y se asignan tareas sin enseñar a los estudiantes el cómo realizarlas (recursos y proceso). El objetivo principal de este estudio es

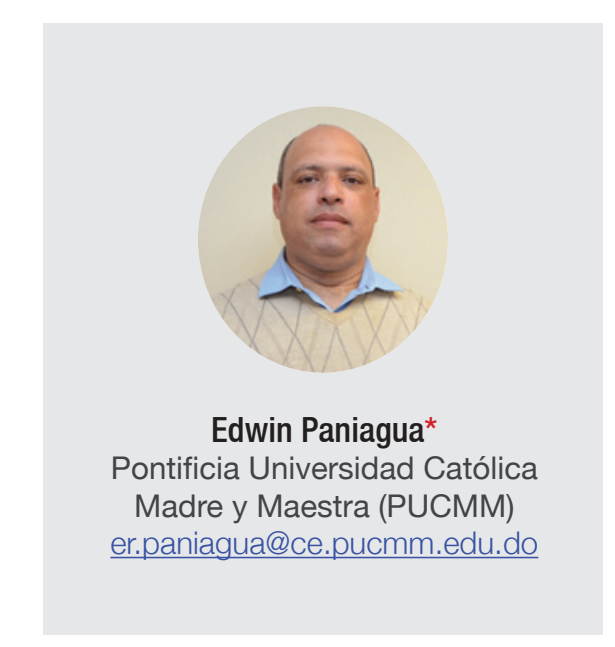
exponer los resultados de la implementación de los enfoques de Aula invertida y TPACK (conocimiento tecnológico, pedagógico y disciplinar), así como la integración de las TAC (Tecnologías para el Aprendizaje y el Conocimiento) para propiciar el aprendizaje de los estudiantes, con la mediación de recursos tecnológicos. En cuanto a la metodología, siguiendo los lineamientos de la investigación-acción, se parte de una evaluación diagnóstica y se programa el diseño de un blog educativo, por equipos. Se provee a los estudiantes de herramientas digitales pertinentes (con tutoriales). Se trabajan los contenidos conceptuales fuera del aula y la clase se concentra en actividades de aprendizaje y aplicación. Entre los hallazgos más destacables, se determina que hay una tendencia general, en los estudiantes, hacia el aprendizaje significativo y autónomo, hacia el desarrollo de competencias, hacia la automotivación y hacia el empoderamiento.

Palabras clave: aprendizaje, aula invertida, competencias, TAC, TPACK

Licenciado en Educación, mención Letras Modernas, magíster en Enseñanza Superior. Docente de la Escuela de Educación de la Pontificia universidad Católica Madre y Maestra (PUCMM). Para contactar al autor: er.paniagua@ce.pucmm.edu.do https://orcid.org/0000-0002-6403-0773 


\section{Abstract}

This project is part of the Educational Innovation course: one hundred students of the Bachelor's Degrees in Mathematics / Chemistry, with an educational orientation. The problem is configured around three fundamental aspects: frequently, even in the virtual modality, the traditional educational model predominates; technology is used as a resource for teaching rather than for learning and tasks are assigned without teaching students how to use the relevant tools (resources and process). Its main objective is to implement the Inverted Classroom and TPACK approaches, as well as the integration of TLK (Technologies for Learning and Knowledge) to promote autonomous learning in students. Regarding the methodology, following the guidelines of action, research the design of an educational blog is programmed, by teams. Relevant digital tools (with tutorials) are provided to students. The conceptual contents are worked outside the classroom and the class concentrates on learning and application activities. Among the most notable findings, it is determined that there is a general trend, in students, towards meaningful and autonomous learning, towards the development of competencies, towards self-motivation and towards empowerment.

Keywords: Flipped Classroom, ICT, Learning, Skills, TAC, TPACK

\section{Introducción}

El presente proyecto se enmarca en la asignatura Innovación Educativa: cuatro grupos (cien estudiantes) de la Licenciatura en Matemáticas / Química, con orientación a la Educación, en la Pontificia Universidad Católica Madre y Maestra (PUCMM), Campus de Santiago, una universidad privada. Se ejecuta en la modalidad virtual (denominada como "enseñanza remota de emergencia” por Marín et al., 2021, p. 9), durante el segundo y tercer ciclo del año 2020. El primer factor que configura el problema es que el modelo educativo tradicional mantiene su predominio, aún en la modalidad virtual: rol pasivo de los estudiantes y énfasis en el resultado. Un segundo elemento es que se usa la tecnología como un recurso para la docencia y no para el aprendizaje. En tercer lugar, se asignan tareas, posteriores a las explicaciones del docente y sin enseñar a los estudiantes a cómo usar las herramientas pertinentes o los procedimientos para realizarlas: se conciben como resultados y no como procesos. Como alternativas, se implementan el aula invertida, el conocimiento tecnológico, pedagógico y disciplinar (TPACK, acorde con Salas, 2021, p. 52) y el uso de las Tecnologías del Aprendizaje y el Conocimiento (las TAC).

Muchos estudios se orientan hacia una reflexión sobre las ventajas y desventajas del aula invertida, del TPACK y de las TAC (Hamdam et al., 2013; Goodwin y Miller, 2013; Bergmann y Sams,
2014; Instituto Tecnológico de Monterrey, 2014; Hernández y Tecpan, 2017; Mejía, 2020; Torres et al., 2021; Constantino y Castillejos, 2020; L'Ecuyer, 2018; Quiroga et al., 2019; Gómez et al., 2016; Díaz et al., 2019 y Paucar, 2019). No obstante, la presente investigación se orienta hacia estudiantes con características muy especiales: educadores en formación, lo cual implica que no solo se trata de ellos como seres individuales, sino de buscar un efecto multiplicador.

En cuanto a la metodología, se programa el diseño de un blog educativo por equipos. Se provee a los estudiantes de herramientas digitales pertinentes (con tutoriales). Se trabajan los contenidos conceptuales fuera del aula y la clase se concentra en actividades de aprendizaje y aplicación. Para la implementación, se sigue el modelo de investigación-acción de Whitehead (relación entre teoría educativa y autodesarrollo profesional, destacado por Latorre, 2005).

El objetivo general de este artículo consiste en exponer los resultados de la implementación del aula invertida, el TPACK y las TAC para propiciar experiencias de aprendizaje en los estudiantes de las Licenciaturas en Matemáticas y Química, con orientación a la Educación, de modo que puedan impactar positivamente a sus futuros estudiantes. En ese sentido, cabe preguntarse: ¿favorecen el aula invertida, el TPACK y las TAC el aprendizaje de los estudiantes de las licenciaturas de Matemática y 
Química, con orientación a la Educación, de modo que también puedan propiciar el aprendizaje de sus futuros estudiantes?

A continuación, se presentarán los conceptos básicos en los que se sustenta esta investigación. Del mismo modo, se describirá la metodología seguida y se analizarán los principales hallazgos. Por último, se presentarán las conclusiones más relevantes, así como las recomendaciones pertinentes y la impresión de varios estudiantes.

\section{Revisión de la literatura}

\subsection{Aula invertida}

Acorde con Hamdam et al. (2013), en sentido general, los docentes se constituyen en la figura central de la práctica áulica y los estudiantes deben tomar apuntes con el fin de ayudarles a realizar las tareas en la casa (2013). Según Goodwin y Miller (2013), para una parte de los estudiantes el progreso puede ser bastante rápido, mientras que, para otros, será lo opuesto. En contraste con esta realidad, ha surgido el modelo o enfoque del aula invertida. Originalmente, esta propuesta fue planteada por dos profesores de Química en una Secundaria estadounidense. En su libro Dale la vuelta a la clase, explican que primero graban un video sobre la clase y se lo envían a los estudiantes. El día de la docencia toman los quince primeros minutos para retroalimentar y activar saberes previos. Luego, en el desarrollo se realiza una práctica guiada o una actividad de laboratorio (Bergmann y Sams, 2014, p. 28). De este modo, la inversión de las actividades favorece que haya más tiempo en el aula para prácticas, conversaciones, realización de proyectos y, por supuesto, para trabajo colaborativo. En la revista Edu Trends, se sintetiza como sigue: "Antes de la clase: los estudiantes revisan los materiales y se preparan para participar en las actividades. Durante la clase, aplican los conceptos y después, evalúan su conocimiento" (Instituto Tecnológico de Monterrey, 2014, p. 5).

En el aula invertida, los docentes se valen de tutoriales (grabados por ellos $u$ otra persona), lecturas, audios, ejercicios... que los estudiantes deben realizar antes de reunirse formalmente. De este modo, llegan con mayores conocimientos previos, aunque también con dudas que les son planteadas a su guía. Los estudiantes deben investigar para familiarizarse con los contenidos conceptuales o teóricos; también deben sintetizar, analizar y discriminar, en el sentido positivo del término. Todo ello antes del siguiente encuentro. De esta manera, el aula se convierte en un espacio para utilizar la información de manera eficiente (competencia), mediante actividades prácticas y de aplicación, en procura de la consolidación del aprendizaje. Por otra parte, sin perder su autonomía, deben interactuar con sus compañeros y con sus docentes. Posteriormente, se asignarán actividades de auto, co y heteroevaluación para reflexionar sobre lo aprendido. En síntesis, el aula invertida implica que los docentes asuman un rol distinto puesto que el protagonismo del aprendizaje recae en cada estudiante. Realmente, ocurre un empoderamiento fruto de la inversión.

La revista Edu Trends lo explica como sigue:

El profesor deja de ser la única fuente de conocimiento. Facilita el aprendizaje a través de una atención más personalizada, así como actividades y experiencias retadoras, que requieren el desarrollo del pensamiento crítico de los estudiantes para solucionar problemas de forma individual y colaborativa (Instituto Tecnológico de Monterrey, 2014, p. 16).

Entre los principales beneficios de este enfoque sobresalen los siguientes: adecuación al ritmo individual de aprendizaje (adaptativo), protagonismo del estudiante, redefinición del rol docente como guía, concepción de la clase como una interacción y el aprendizaje como un proceso, favorecimiento del aprendizaje autónomo, por lo cual despierta la motivación y el interés de los estudiantes. Y cabe destacar que este enfoque requiere la redacción de consignas claras y de un acompañamiento docente constante (por diversas vías), que incluya respeto por las ideas de cada estudiante, guía para los procedimientos y, en especial, recursos para el proceso.

Muchos docentes siguen enfocando su actividad hacia un resultado y omiten el proceso imbricado: el cómo y el para qué quedan obliterados en el qué. En adición, tanto estudiantes como docentes pueden tener la sensación de que no han utilizado provechosamente el tiempo. De ahí que implementar 
la metodología o el enfoque de aula invertida venga, precisamente, a revertir o a potenciar el proceso de enseñanza aprendizaje, orientándolo hacia actividades de aprendizaje y capitalizando al máximo el tiempo dentro y fuera del aula.

\subsection{Tecnological Pedagogical Content Knowledge (TPACK)}

En conexión directa con el último enunciado del apartado anterior, es preciso reflexionar sobre lo que implica el acrónimo TPACK (Tecnological Pedagogical Content Knowledge: Conocimiento Tecnológico y Pedagógico del Contenido). Se refiere a un modelo que "reconoce la importancia de los tres componentes fundamentales: contenido, pedagogía y tecnología, centrando el foco en las múltiples interacciones que existen entre ellos" (Posada, 2013). En esas escuetas palabras se conforma un campo semántico fundamental para la práctica educativa y, en específico, áulica: contenido, pedagogía y tecnología. En esencia: el dominio del contenido, de la pedagogía del contenido y de la tecnología pedagógica o didáctica para ese contenido.

EI TPACK, cuya formulación o síntesis Gómez (2015, p. 186) se la atribuye a Punya Mishra y Mattew J. Koehler, vincula esas tres dimensiones del acto educativo en un mismo plano. Resulta obvio plantear que no es posible enseñar lo que no se sabe o lo que se desconoce, pero se apresuraría alguien que afirme que puede enseñar todo lo que sabe. El saber es epistemológico, pero la enseñanza es metodológica, pedagógica y didáctica. Y, sobre todo en la actualidad, es evidente que tiene un componente tecnológico.

\section{Figura 1.}

\section{Imagen que explica el TPACK}

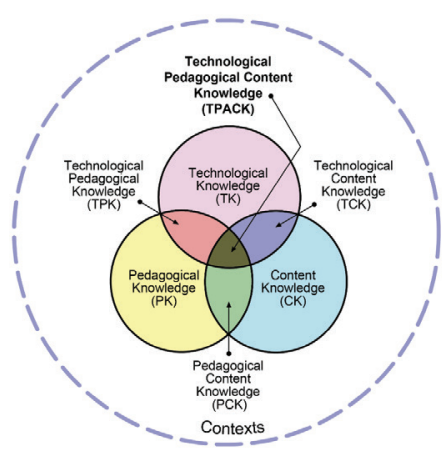

Del diagrama anterior se infiere que las tres variables constituyen la intersección del aprendizaje, pero, a su vez, se intersectan en otros puntos. Tomando como base la traducción de Posada (2013), se citan los siguientes elementos o aspectos (en general):

a) Conocimiento pedagógico del contenido. Además de lo conceptual, quien enseña debe saber organizar, graduar o dosificar, segmentar, planificar, vincular y evaluar el contenido.

b) Conocimiento tecnológico del contenido. Los docentes no solo deben dominar el contenido, sino que deben utilizar una tecnología adecuada a su enseñanza.

c) Conocimiento tecnológico pedagógico. Los docentes deben conocer a profundidad las ventajas y los límites de los recursos tecnológicos y de las estrategias metodológicas, didácticas y pedagógicas que implementan, para emplearlas de manera provechosa.

EI TPACK, pues, implica que los docentes provean a sus estudiantes de herramientas tecnológicas pertinentes e, incluso, de los tutoriales y las guías para que las utilicen provechosamente.

\subsection{Recursos tecnológicos (TIC y TAC) y desarrollo de competencias}

Es de rigor destacar que ni el aula invertida ni el TPACK se enfocan en la tecnología como objetivo principal: "No consiste en un cambio tecnológico: únicamente aprovecha las nuevas tecnologías para ofrecer más opciones de contenidos a los estudiantes y, lo más importante, redefine el tiempo de clase como un ambiente centrado en el estudiante" (Bergmann y Sams, 2013, p. 17). No solo se trata de más opciones de contenidos, sino de propiciar mayores y más variados espacios de aprendizaje (incluyendo contenidos, procedimientos, herramientas, colaboración, interdependencia, evaluación...):

Cada estudiante es el centro de interés en el proceso educativo puesto que son quienes descubren y regulan su aprendizaje, según a la experiencia e intereses que se potencializan en diferentes recursos ofrecidos por las TIC. El estudiante, desde la modificación cognitiva y 
actitudinal, desarrolla aprendizajes significativos por medio de estrategias de interacción, participación, argumentación y la colaboración. La enseñanza reflexiva del docente puede ser adaptativa, con utilización de estrategias de interacción social, encaminadas a facultar en el estudiante la autonomía y la organización de la información (metacognición). El andamiaje producido por el apoyo y la construcción de conocimiento con el otro, permite generar una motivación intrínseca a través de la facilitación y orientación del aprendizaje por parte del docente (Padilla et al., 2014, p. 277).

Ello implica una evolución desde el concepto de aprendizaje significativo (basado en su carácter no arbitrario y su no literalidad, según Ausubel, 2002), ya que no se trata de una realidad intrapersonal o psicológica, sino que la intención es que el estudiante utilice el conocimiento construido. "Las tecnologías de la información y comunicación en la educación superior representan los nuevos entornos de aprendizaje y, por su impacto en la educación, son desarrolladoras de competencias necesarias para el aprendizaje y generadoras de habilidades para la vida" (García et al., 2017, p. 3). En igual dirección, se expresan Villa y Poblete:

Una parte importante de la innovación pedagógica se apoya en el uso didáctico y pedagógico de estas tecnologías tanto por parte del profesorado como de sus alumnos. Esta utilización ayuda a crear nuevos espacios virtuales que favorecen la autonomía de alumnos y multiplican las posibilidades educativas de interacción y creación (2007, p. 12).

Los planteamientos anteriores evidencian la clara vinculación entre el aula invertida, el TPACK, las TAC y el empoderamiento estudiantil, imprescindible tanto para el aprendizaje significativo y autónomo como para el desarrollo de las competencias. "El enfoque de formación basado en competencias implica que el aprendizaje comienza a ser el centro de la educación, más que la enseñanza" (Tobón, 2006, p. 14). Se trata, en síntesis, de que cada estudiante trabaje, antes de la clase, los contenidos conceptuales (mediante documentos pertinentes, preferiblemente con recursos que le permitan una evaluación). Aquí sería importante destacar que, si deben realizar una actividad con una herramienta específica, su docente les provea no solo de la misma, sino que los oriente sobre su uso eficiente. En el aula, se debe trabajar con actividades de aplicación y de aprendizaje, con su respectiva evaluación formativa y acompañamiento. Los docentes son realmente guías:

Otra recomendación es la de crear nuevos entornos pedagógicos, que van desde los servicios de educación a distancia hasta los establecimientos y sistemas virtuales de enseñanza superior, capaces de establecer sistemas de educación de alta calidad, favoreciendo el progreso social, económico, la sostenibilidad, así como otras prioridades sociales importantes. Además, la figura del maestro será ahora la del facilitador del aprendizaje (García et al., 2017, p. 303).

Todo lo anterior favorece la evolución de las tecnologías de la información y la comunicación (TIC) a las tecnologías para el aprendizaje y la comunicación (TAC). Al respecto, González et al. (2020) plantean que la tecnología se ha convertido en un recurso primordial en el aula. $Y$ destacan que este avance, es decir, utilizar tecnología específicamente educativa, favorece la implementación de metodologías activas en el aula. La cita precedente cobra mayor relevancia en el contexto de la pandemia, en el cual se hizo necesaria la migración de todo el sistema educativo hacia plataformas digitales. De ahí que, el uso de herramientas propias del ámbito académico haya producido una especialización de la tecnología, tanto por parte de docentes como de estudiantes. En ese sentido, por ejemplo, en las matemáticas, ya no solo se trata de utilizar herramientas de presentación como Power Point, sino de aprovechar los beneficios que ofrecen programas o aplicaciones propias del área, como Geogebra.

Y, en adición, se ha llegado a destacar que este proceso tampoco se detiene en las TAC, sino que sigue desarrollándose: "A partir de estas mediaciones tecnológicas (TAC), la escuela promueve en los estudiantes una postura de crítica y análisis, constructiva y responsable, difundidas o socializadas mediante las TEP, a saber, las tecnologías de empoderamiento y participación" (Latorre et al., 2018, p. 37). 


\section{Metodología}

Esta investigación abarca un período de dos ciclos académicos y su universo lo constituyen cien estudiantes de la Licenciatura en Matemáticas/ Química, con orientación a la Educación, en la asignatura Innovación Educativa. La metodología seguida fue orientada por la investigación-acción. Como técnica, se utilizó la observación estructurada, mediante lista de cotejo / rúbrica (validada por un docente de Informática Educativa, de la universidad). Como instrumentos, se aplicaron dos cuestionarios que contenían preguntas cerradas y abiertas (el primero como evaluación diagnóstica y el segundo para que el docente recibiera retroalimentación sobre la percepción de los estudiantes sobre el desarrollo de la asignatura, al final).

Para el presente estudio se siguió el modelo de investigación-acción planteado por Whitehead (Latorre, 2005, p. 38), cuyas fases son las siguientes: sentir e identificar un problema, imaginar y poner en práctica la solución, evaluar los resultados de las acciones y modificar la práctica a la luz de los resultados.

En cuanto a la primera fase sentir e identificar un problema (diagnóstico), se utilizan preguntas orales y escritas, estas últimas en la plataforma de la universidad, tanto sobre el contenido de la asignatura como del manejo de la tecnología propia del proceso de enseñanza-aprendizaje, mediante un formulario de Google.

En sentido general, los estudiantes manifestaron que, a pesar de tener más participación que antes en sus clases en la universidad, todavía mantenían un rol pasivo y que predominaban las actividades realizadas por el docente. También destacaron que la mayoría de sus docentes no implementaban el aula invertida. Del mismo modo, indicaron que habían escuchado sobre algunas herramientas tecnológicas, pero que la mayoría de ellas solo había sido utilizada por sus docentes o espontáneamente por ellos, pero no de manera sistémica ni sistemática.

En lo referente a la fase de solucionar el problema, el docente toma la decisión de implementar el aula invertida, el TPACK y formalizar el uso de las TAC. Para ello, se conforman (libremente) los equipos para el diseño de un blog educativo (un máximo de cuatro estudiantes). Se presenta un cronograma con las actividades a realizar durante la asignatura, incluyendo los vínculos a los recursos y documentos digitales que se emplearían. Se distribuyen las actividades de evaluación, las horas y canales de tutorías.

En adición, se muestran las dos plataformas que se utilizarían de manera recurrente para la docencia: Microsoft Teams y la versión de Moodle de la universidad. Por último, se crea un grupo en WhatsApp. En consonancia con lo anterior, se trabajan los contenidos conceptuales fuera del aula (antes de cada clase, de manera asincrónica) y en cada clase se revisan las respuestas de los estudiantes (a través de organizadores gráficos, videos e infografías) y se auto y coevalúan. A continuación, se proporciona el espacio para que se reunieran en los canales de Teams para ir diseñando una actividad en el blog. El docente acompaña y monitorea a cada equipo (evaluación formativa) y al final, se realiza una plenaria del progreso de la actividad. En el cierre de la clase se proponen vínculos y enlaces, tanto para el contenido conceptual como el procedimental del próximo encuentro. De igual manera, se proporciona una plantilla para planes de clase. Con base en ella, se dialoga con el docente para preparar la clase simulada que se ejecutará de manera simulada y sincrónica, utilizando como herramienta o recurso principal el blog creado por los equipos.

En lo vinculado con el aspecto evaluación, se dialoga sobre la rúbrica que se utilizaría en la evaluación del proyecto, la cual fue elaborada por el docente de la asignatura y validada por otro docente del área de Tecnología Educativa, de la Escuela de Educación. Además, se establece el plazo para la presentación de la primera parte del proyecto y de la fecha final. En total, se estructuraron cinco tareas (todas vinculadas con el blog), un control de lectura (sobre un artículo académico relacionado con innovación educativa y formación docente), la presentación parcial (dos unidades) y final (dos unidades más) del blog, así como la realización de una clase simulada, en la que se espera el uso de los recursos tecnológicos estudiados y del blog creado. En general, la evaluación del progreso se trabaja desde el inicio y en cada actividad, mediante las tareas, el progreso en el diseño del blog, así 
como en la preparación y ejecución de la clase simulada. El docente acompaña a los equipos y a los estudiantes, de manera individual, mediante momentos específicos en la llamada (sincronía) como en tutorías personales y grupales (asincrónica), también por medio de WhatsApp. Los resultados arrojan que, progresivamente, los estudiantes se empoderan tanto de los contenidos conceptuales como de los procedimentales. Entregan sus asignaciones ajustándose a los criterios indicados. Planifican, diseñan y ejecutan tanto el blog como la clase simulada implementando la metodología de aula invertida, el TPCK y las TAC, así como la gamificación y la interdisciplinariedad.

En la fase de modificar la práctica a la luz de los resultados, se propone mantener una reflexión y conversación constantes, así como una retroalimentación formal, por parte de los estudiantes sobre el proceso y el resultado, a través de un formulario de Google. Luego de ello, el docente recoge las informaciones recibidas, las analiza y realiza los ajustes en el cronograma de la asignatura y en la plataforma de la universidad.

Cabe destacar que la asignatura se orienta hacia estos resultados de aprendizaje:

a) Diseñar un proyecto de innovación educativa para impulsar la mejora del proceso de enseñanza y aprendizaje de una disciplina específica (diseño de un blog).

b) Analizar diversas perspectivas teóricas construidas alrededor del concepto y las características que definen la innovación educativa, a fin reconocer su importancia para un efectivo desarrollo del currículo (control de lectura y asignaciones o tareas).

c) Desarrollar una práctica pedagógica en el aula con la utilización de estrategias y actividades innovadoras, para favorecer en los estudiantes el desarrollo de una competencia específica (clase simulada).

d) Planificar una clase modelo con la utilización de entornos virtuales de aprendizaje, para potenciar el uso de las TAC como herramientas para el aprendizaje (clase simulada).

\section{Resultados}

En cuanto a la primera fase sentir e identificar un problema (diagnóstico), se utiliza un formulario de Google para determinar su nivel de conocimiento sobre herramientas específicas o su uso. Las opciones se concentran en herramientas tecnológicas para la docencia y, en menor grado, se refiere a estrategias que se emplean en el proceso enseñanza-aprendizaje. En este sentido, lo que se pretende es determinar el conocimiento que poseen los estudiantes de estos elementos, ya sea porque los conocían de primera mano o porque habían escuchado hablar sobre ellos.

Como se aprecia en la figura 2, entre el $90 \%$ y el $100 \%$ de los estudiantes había escuchado sobre herramientas y estrategias como Kahoot, blogs y la resolución de problemas. En segundo lugar, oscilando entre un 40 y un 60\%, indicaron el aula invertida, los mapas mentales, la realidad virtual, la gamificación y Quizizz. En tercer lugar, por debajo del 20 y el 30\%, señalaron Padlet, Podcasts, Powtoon, Mentimeter y la realidad aumentada. Por último, manifestaron que desconocían herramientas como Edpuzzle, Vizia, Visme, Flipgrid, Infogram, Mindmeister, Genial.ly e Infogram, así como el TPACK y el movimiento Makers. Así se muestra en la figura 2:

\section{Figura 2.}

Conocimiento tecnológico y pedagógico de los estudiantes

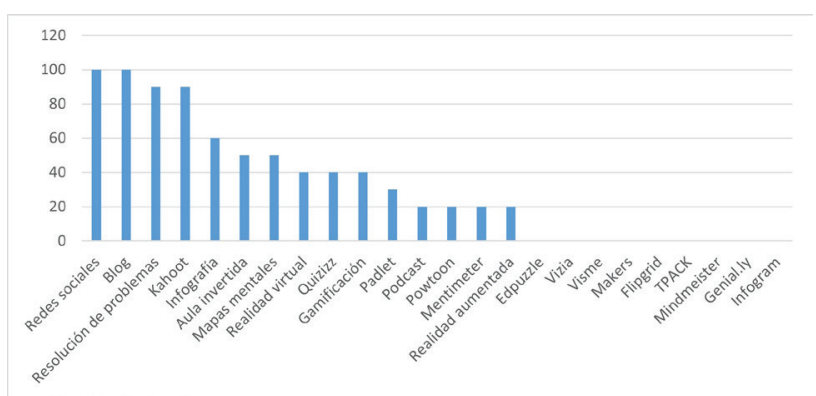

Ahora bien, cuando se les realiza la misma pregunta, pero orientada a si utilizan esas herramientas y estrategias, en sus prácticas, el resultado varía inversamente en los campos donde habían contestado con mayor porcentaje. Dicho de otra manera, admiten que conocen esos recursos y estrategias, pero que no los emplean cuando asumen el rol docente. Aducen que, básicamente, 
se concentran en explicar los contenidos lo mejor posible. Y, a pesar, de que muchos conocen o tienen blogs, no los utilizan para la docencia (dicen que los tienen porque los crearon para el trabajo final de alguna asignatura, por ejemplo, pero en cuanto a la estructura básica y nunca trabajaron en su diseño). Igual ocurre con las redes sociales: no las integran al proceso de enseñanza-aprendizaje. Así se manifiesta en la figura 3:

\section{Figura 3.}

Implementación de estrategias, metodologías y recursos por parte de los estudiantes

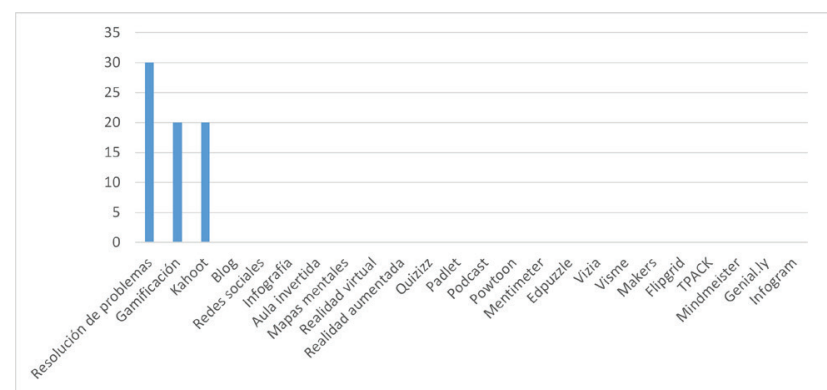

En lo referente a la fase de solucionar el problema, de manera general, se trabajan los contenidos conceptuales fuera del aula, mediante videos, artículos académicos, documentos en sitios Web, plataformas digitales sobre los cuales se debe entregar un producto (videos con preguntas incrustadas, videos animados, videos en Flipgrid, mapas conceptuales, infografías...). Se trabajan simultáneamente los contenidos conceptuales y los procedimentales (usualmente, mediante varios tutoriales), así como el desarrollo de las competencias. Incluyendo tutoriales (curados) sobre el uso de esas herramientas. Todo ello antes de la clase. El docente revisa dichas asignaciones previo a la sesión debido a que debían entregar sus asignaciones con un día de antelación.

Lo anterior tiene un doble impacto positivo: en primer lugar, debe dominar el contenido y la herramienta para trabajarlo; en segundo lugar, en ambos grupos, la entrega de estas tareas pasa de casi un 50\%, en la primera, hasta 90\% - 100\%, en las últimas tres. Dicho de otra manera, los participantes trabajan el contenido de la clase, antes de llegar a ella; también se familiarizan con una herramienta tecnológica (distinta cada vez) y la cantidad de entrega de las tareas se incrementa, paulatinamente, hasta el 100\% (aumenta en casi un 40\% en la segunda entrega). Así lo evidencia la figura 4:

\section{Figura 4.}

Incremento de la entrega de la asignación previa

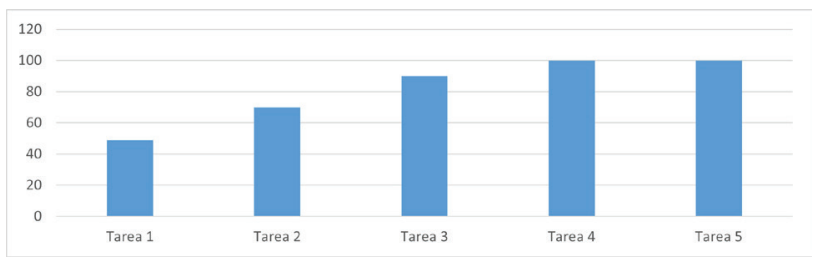

En el inicio de la clase, se realiza una retroalimentación general delos contenidos conceptuales, socializando los trabajos elaborados por cada estudiante. Surge el mínimo de dudas. Como fortalezas, en el $80 \%$ de los trabajos (incluidas las presentaciones preliminar y final del blog) se exhibe un dominio conceptual, suficiente para cumplir con las evidencias de aprendizaje. En cuanto a lo procedimental, el 100\% de los estudiantes manifiesta que les resultan útiles los tutoriales aportados por el docente e, incluso, los que contenían algunas de las páginas visitadas. En este sentido, solo un 20\% se comunica con el docente durante el proceso de elaboración de las tareas para requerirle su ayuda. Como resultado, los estudiantes se empoderan de su proceso de aprendizaje.

En lo referente al desarrollo de la clase, luego de la retroalimentación y de la activación de saberes previos, así como de la motivación del tema del día, los estudiantes se reúnen en sus equipos para diseñar una actividad de aplicación que se pueda integrar en el blog que deben realizar. Se les orienta para que integren la herramienta utilizada en la tarea. Para ello, el docente crea un canal para cada equipo, en Microsoft Teams.

En él, se comparten los archivos y documentos necesarios para este momento. También, recursos importantes (que están en el cronograma) disponibles en la adecuación de Moodle de la universidad y en el chat de WhatsApp. Ello permite que los estudiantes puedan acceder desde diversos dispositivos, así como descargar los recursos y los documentos.

El docente visita cada una de las salas creadas o de los canales para orientar los procesos. Los estudiantes comparten la pantalla y proyectan tanto 
la herramienta que trabajan como su inserción en el blog. De este modo, el centro de la clase lo ocupan las actividades de aprendizaje y de aplicación. Es preciso señalar que cuando surge una duda, se motiva para que otra persona del equipo conteste, antes que el docente. Por otra parte, al trabajar la aplicación en el aula se reduce la cantidad de tutorías generales o particulares (por equipo o por estudiantes). No más de tres o cuatro personas (individualmente) o uno o dos equipos contactan al profesor para aclarar dudas, fuera de clase: la mayoría se resuelven durante el encuentro virtual (la videollamada).

En adición, siempre se motiva a que cualquier inquietud sea manifestada de manera pública, en la medida de lo posible, para que sirva de soporte a los demás integrantes. Al final, en una plenaria, un representante de cada grupo presenta el resultado del trabajo de su equipo. Entre un $70 \%$ de los casos se completa el proceso de integrar la herramienta y la actividad al blog, durante la clase. En cuanto al porcentaje restante, se programa otro encuentro (a lo interno del equipo) para finalizar antes de la siguiente sesión. En estos casos, el porcentaje anterior aumenta a un 80\%. Durante el cierre, se emplea una herramienta tecnológica para confirmar lo aprendido y explorar cómo se han sentido los estudiantes con relación a su propio trabajo y a la clase. Para ello, los recursos más utilizados son Mentimeter, WordArt, Google Forms y Microsoft Forms. Con frecuencia, los estudiantes expresan satisfacción con lo aprendido y con el desarrollo del proceso de enseñanza-aprendizaje. Con lo descrito, con relación a las clases, un resultado destacable es que se fomenta una participación, prácticamente, total de los estudiantes (antes, en el inicio, en el desarrollo y en el cierre de la clase); en consecuencia, ellos se convierten en los protagonistas de su aprendizaje. El docente, por su lado, pasa a fungir como un guía del proceso. Así se refleja en la figura 5:
Figura 5.

\section{Empoderamiento de los estudiantes}

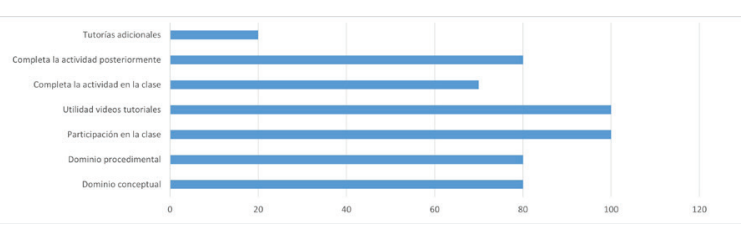

En otra de las actividades principales de la asignatura, los estudiantes planifican y ejecutan una clase, simulando que están en un contexto real de un centro de educación secundaria. Cada equipo imparte una clase, previa planificación y conversación con el docente (90\%), implementando las metodologías o enfoques / metodologías de aula invertida y TPACK, además de las TAC, en sus respectivas áreas.

En el $70 \%$ de los casos, los practicantes que fungen como docentes, envían una asignación previa con al menos uno o dos días de antelación, que son completadas y valoradas por sus compañeros como positivas, interesantes y prácticas. El resto suele consistir en actividades enviadas con poco tiempo de antelación y con una valoración menor a la expresada con anterioridad, en todos los renglones. En general, motivan a sus compañeros a completar las actividades, a través del grupo de WhatsApp. En el inicio, retroalimentan la asignación y se presentan los contenidos, las competencias y los indicadores de logro que se proponen trabajar ese día, en el 100\% de los casos. En esta parte, la mayoría utiliza herramientas de gamificación, redes sociales, videos con preguntas incrustadas (Edpuzzle, H5p, Vizia...) e infografías. Por otro lado, todos los recursos y actividades se realizan con base en el blog. El desarrollo se concentra en actividades de aplicación de los contenidos conceptuales, en resolución de problemas y apelando al uso de herramientas digitales propias de su disciplina (incluyendo recursos de apoyo para los procedimientos y el uso de las herramientas), seguido de una plenaria, en el $80 \%$ de los casos. El porcentaje restante se dedicó exclusivamente a los contenidos conceptuales. Para el cierre, la mayoría (90\%) utiliza gamificación (Sgame.dit.upm. es, Genial.ly, Quizizz, Kahoot, Cerebriti, WordWall, 
Educaplay...) para retroalimentar la clase. Cabe destacar que, en ocasiones, se planifica utilizar una de estas herramientas para el cierre, pero debido a inconvenientes con el manejo del tiempo no se utilizan.

Al final de cada clase, el facilitador de la asignatura propicia la auto y la coevaluación mediante la herramienta Mentimeter, en la cual están resumidos los criterios de la ficha de observación, elaborada por los especialistas de la Escuela de Educación de la universidad. Luego, el docente se reúne con cada equipo para analizar la clase y conversar sobre la heteroevaluación, con base en los criterios establecidos.

Como se infiere, los estudiantes-practicantes evidencian dominio del aula invertida, del contenido disciplinar, de la pedagogía y, en consecuencia, del TPACK. También orientan la clase hacia actividades de aplicación y de resolución de problemas. Fomentan la participación y el protagonismo de sus alumnos y, en adición emplean, la gamificación y la evaluación, además, de planificar la clase acorde a sus momentos.

Con ello, sustituyen el modelo educativo o didáctico tradicional y tienden hacia corrientes pedagógicas que fomentan la construcción del conocimiento por parte del estudiante, así como hacia la integración de la tecnología. Con ello, como se especificó al inicio del presente artículo, no solo se cambia de paradigma en cuanto su rol de estudiantes, sino en cuanto a su presente y futura práctica docente, tanto por el enfoque en el que se coloca al estudiante como protagonista de su proceso de aprendizaje, como a la integración dinámica de la tecnología. Esto se resume en la gráfica 6:

\section{Figura 6.}

\section{Análisis de los resultados de la clase} simulada

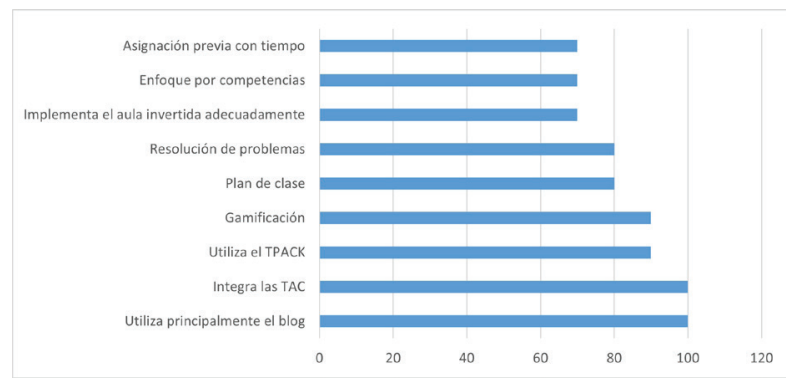

En lo referente a la presentación preliminar y final del blog, con base en la rúbrica utilizada, los resultados se presentan a continuación. En cuanto a la estructura, organización y disposición de los elementos (funcionalidad y usabilidad, navegabilidad, accesibilidad, visualización, legibilidad...) los blogs creados por los estudiantes oscilan entre un 50 y un 90\% de adecuación, entre una etapa y otra. En lo referente al diseño (motivación y dinamismo que genera, interacción, participación, creatividad, diseño atractivo y ágil) ocurre una oscilación similar: 60-80\%. Igual sucede con la orientación hacia el usuario (el énfasis de las actividades recae sobre el alumno-usuario, atención a los estilos de aprendizaje, adecuación al destinatario....): 50-80\%. Al principio casi sobresale el aspecto de transmisión de conocimientos, pero al final se destaca la multiplicidad de formatos de los textos, así como de las actividades que implican una doble vía.

Por otra parte, en cuanto a los aspectos pedagógicos y metodológicos (correspondencia con el Diseño Curricular, orientado hacia el desarrollo de competencias, aprendizaje basado en problemas, basado en retos, autónomo, significativo, interdisciplinariedad, vinculación con la vida y propiciar el aula invertida y el TPACK) los estudiantes avanzan, en el blog, desde un 40 hasta un $80 \%$. Ello debido a que la base de su trabajo se orienta por las competencias curriculares del Nivel Secundario, plantean problemas vinculados con la cotidianidad, integran actividades que implicaban la interdisciplinariedad (por ejemplo, aplicando la matemática a la astronomía, a la medicina y al ambiente). En adición, evidencian dominio del contenido, de su pedagogía y de su tecnología (TPACK), así como presentan una estructura y organización de los contenidos que favorece el enfoque por competencias y el aula invertida, debido a que colocan la parte conceptual al inicio del blog y, luego, se concentran en las actividades descritas.

Por último, en relación con las TAC, los alumnos parten de un 65 y llegan a un 95\% debido a la integración dinámica de tutoriales, aplicaciones, plataformas, infografías, gamificación, videos, películas, creación de contenidos, redes sociales $\mathrm{y}$, en menor escala, realidad virtual y aumentada. 
Paulatinamente, los estudiantes amplían su banco de recursos y herramientas digitales mediante una variedad propia de los procesos pedagógicos y didácticos, en general, pero también de los procesos específicos de los contenidos disciplinares, es decir, de sus asignaturas (Geogebra, simuladores...). Así se manifiesta en la figura 7:

\section{Figura 7.}

\section{Presentación preliminar y final del blog}

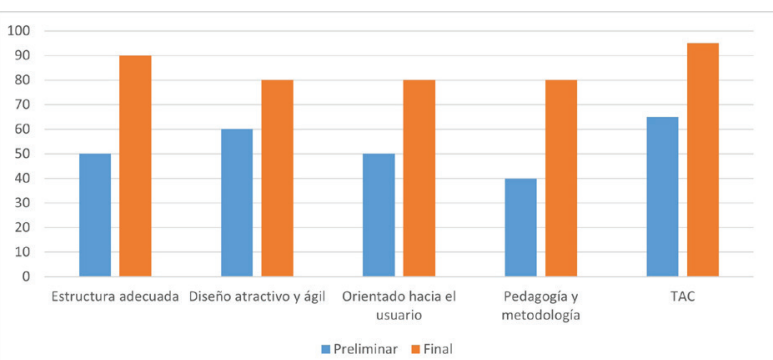

En otro tenor, en lo vinculado con el aspecto evaluativo de la asignatura, en total, se estructuran cinco tareas (todas vinculadas con el blog, realizadas por la mayoría), un control de lectura (sobre un artículo académico relacionado con innovación educativa y formación docente), la presentación parcial (dos unidades) y final (dos unidades más) del blog, así como la realización de una clase simulada, en la que se espera el uso de los recursos tecnológicos estudiados y el uso del blog creado. En promedio, el $70 \%$ de los estudiantes obtiene una calificación de A; el 25\%, B y el 5\% C, en consonancia con los instrumentos de evaluación referidos.

En cuanto a la fase de Evaluar la implementación de la estrategia, como ya se ha señalado, se evidencia en los estudiantes un dominio disciplinar, pedagógico y tecnológico (TPACK y TAC), así como la orientación hacia el enfoque por competencias, la resolución de problemas y la implementación del aula invertida, con un acierto de un 70-75\%. Antes de pasar al siguiente punto, para que se tenga una idea más clara de los medios utilizados, se enumeran la mayoría de los recursos TAC empleados en la asignatura, tanto por el docente como por los estudiantes:

MOODLE, Microsoft Teams, WhatsApp, OneDrive (videollamada, materiales y tutorías), Infogram, Genially, Pinterest y Canvas (infografías), H5P, Edpuzzle y Vizia (integración de preguntas a videos), Flipgrid (grabación de videos comentando contenido de la clase y fomentar la participación), Powtoon, Animaker y Animoto (para realizar videos animados), Mozaweb (contenidos digitales en 3D y realidad virtual), Walla me (realidad aumentada), Anchor (grabación de audios), Mentimeter (nube de palabras para retroalimentar y para coevaluar), Word Art (nube de palabras con figuras), YouTube (tanto para tutoriales como para contenido de la clase), especialmente, por parte del docente (clase y asignaciones).

En adición a las anteriores, también se recurrió a las siguientes: Genial.ly, Wordwall, Flippity, Educaplay, juegosarcoiris.com, mobbyt.com, sgame.dit. upm.es y Cerebriti (gamificación), Google forms, Kahoot y Quizziz (evaluación), Mindmap Maker, Lucid Chart y Mindmeister (mapas mentales), Padlet (trabajo colaborativo), Facebook, Instagram, Twitter... (para compartir contenidos y trabajar con retroalimentación mediante encuestas), diversas aplicaciones descargadas de Google Play, AppSorteos (para fomentar la participación mediante una ruleta digital), Filmora (grabación de un trailer para presentar una clase), Geogebra (Software especializado en el área de Matemática), así como varios simuladores y tablas periódicas interactivas (en 3D) en la asignatura de Química. En cuanto a las plataformas elegidas por los estudiantes para el diseño del blog, están las siguientes: Blogger, Word Press, Wix, Google Sites, Simple Sites, Webnode y Jimdo.

En ello, se aprecia un empoderamiento que se refleja en la comparación de los gráficos 2 y 3 con 5, 6 y 7 , en los cuales se evidencia que los estudiantes pasaron de haber escuchado sobre herramientas tecnológicas, enfoques pedagógicos y metodologías, a un dominio igual o superior al 70\% de los mismos.

En relación con la última fase: Modificar la práctica a la luz de los resultados, se recibe la retroalimentación de los estudiantes, a través de un formulario en Google Forms (la mayor parte de la valoración de los estudiantes se detallará en el próximo apartado) y se realiza una reunión con el especialista en Informática Educativa de la Escuela de Educación (coevaluador de los blogs). En ese sentido, las sugerencias o modificaciones más relevantes que surgen son las siguientes: a) actualizar algunos 
vínculos que presentan problemas, b) reorganizar los contenidos, tanto en el cronograma, como en la plataforma Moodle, c) utilizar más tiempo del estipulado para retroalimentar algunos contenidos conceptuales, al inicio de la clase, d) continuar explorando e incorporando nuevas herramientas digitales, e) reflexión sobre el exceso de tiempo que requirieron varias asignaciones, f) repensar las estrategias de acompañamiento para lograr que la inmensa mayoría desarrolle las competencias de la asignatura y asuma lo propuesto, y g) integrar herramientas y actividades de realidad aumentada y virtual, las cuales se presentaron y analizaron, pero no se tuvo el tiempo para explorarlas y adecuarlas apropiadamente.

\section{Discusión y conclusiones}

El objetivo general de este artículo consistió en exponer los resultados de la implementación del aula invertida, el TPACK y las TAC para propiciar experiencias de aprendizaje en los estudiantes de las Licenciaturas en Matemáticas y Química, con orientación a la Educación, de modo que puedan impactar positivamente a sus futuros estudiantes.

En este sentido, como cada estudiante trabaja directamente con los contenidos conceptuales y procedimentales, antes de clase, se evidencia una apropiación de ellos, así como facilidad para su aplicación. En adición, los estudiantes expresan que están aprendiendo de una manera muy sutil e interesante, con autonomía, lo cual incrementa su entusiasmo. Todo lo anterior deviene en un empoderamiento que les resulta estimulante, a decir de ellos. Una de las estudiantes lo expresa así:

Le doy a esta clase la valoración máxima que pueda haber, es decir, el total: 100\%. Esta asignatura me ha enseñado que, además de facilitar el aprendizaje, el docente lo hace de manera tan dinámica, que aprendemos sin saber que lo estamos haciendo.

En este comentario ella se refiere a que el docente trabaja para "facilitar el aprendizaje"; es decir, que su rol es activo y que el profesor de la asignatura es, literalmente, un facilitador. Cada docente pasa de ser "the sage on the stage" a ser "the guide on the side" (King, 1993, p. 30). El docente pasa de ser el sabio (la estrella, el protagonista) a ser un guía. Por otra parte, se destaca que el interés que se despertó por aprender llegó a un punto de ser natural y fluido: "Sin saber que lo estamos haciendo". En similar tesitura se expresa otra estudiante:

La metodología es excelente, las clases son muy dinámicas y entretenidas, en ningún caso monótonas y cansonas. Considero que los recursos utilizados por el docente son casi inmejorables, cubren casi cualquier área del saber no solo en Matemáticas.

La aplicación del aula invertida, pues, no solo genera en los estudiantes un aprendizaje significativo y autónomo, sino que los interesa, propicia el desarrollo de las competencias esperadas y deviene en un empoderamiento tanto conceptual como procedimental y actitudinal. Estos resultados contrastan con la resistencia esperada al cambio: "Destacaremos la reticencia que pueda mostrar el alumnado, que puede optar por preferir el método tradicional, negándose así a abandonar su zona de confort" (Aguilera et al., 2017, p. 263).

Por otra parte, al final, se les pregunta a los estudiantes (Google Forms) sobre su dominio, en relación con los resultados de aprendizaje de la asignatura, en una escala del 1 al 5 (siendo este último el grado máximo). Responden entre un 85 y 90\% en el nivel 5 (máximo) y el resto, en el nivel 4. Se colige que sienten que dominan los contenidos, fruto de su contacto directo con ellos.

En cuanto a asumir el enfoque TPACK para propiciar el aprendizaje de los estudiantes, cada herramienta que los estudiantes deben utilizar cuenta con vínculos a varias aplicaciones (por ejemplo, en el caso de las infografías: Infogram, Canva, Genial. ly, Picktochart...), así como tutoriales que faciliten su uso eficiente.

Cada uno de los recursos utilizados por el maestro fueron trabajados de manera excelente, ya que me ayudaron a aprender a utilizar diversas estrategias para favorecer tanto mi aprendizaje como el de mis futuros estudiantes.

En el comentario anterior sobresalen recursos y estrategias, así como "mi aprendizaje" y el de "mis futuros estudiantes"; es decir, se reflexiona sobre el aprendizaje propio y, además, sobre el de los futuros estudiantes de este docente en formación. 
Dicho de otra manera, se percibe un dominio no solo conceptual, sino pedagógico, didáctico y metodológico. Cada contenido conceptual se trabaja antes de la clase con diversos tipos de documentos, requiriendo el uso de una herramienta digital y proporcionando varias alternativas de ellas, incluidos distintos tutoriales sobre su uso. Dicho de otra manera, a los estudiantes no solo se les propone el abordaje de unos contenidos, sino que se les proporciona opciones de herramientas para hacerlo, con sus respectivos tutoriales.

Ahora bien, no faltan docentes que expresan su temor a que el medio se confunda con la finalidad: "Si no se extraen informaciones útiles o válidas, puede suponer una visión parcial de la realidad, que no llega a entrar en la estructura de conocimientos del estudiante, siendo una pérdida de tiempo" (Torres et al., 2021, p. 107). En una tesitura similar, se expresan otros autores: "Pero su uso se limitó a una práctica procedimental" (Constantino y Castillejos, 2020, pp. 467-468). También está la aseveración frecuente de que los jóvenes carecen de los criterios para valorar la información que se les presenta, a pesar de sus competencias digitales o técnicas (L'Ecuyer, 2018).

Muy por el contrario, en el presente estudio, los estudiantes demuestran un dominio de los contenidos conceptuales y procedimentales que, incluso, se refleja en los actitudinales y en las emociones que manifiestan. El Ministerio de Educación de la República Dominicana establece que los contenidos son mediadores de aprendizaje significativo y se clasifican como conceptuales (teóricos), procedimentales y actitudinales (interiorización de los anteriores y manifestación de una conducta consecuente), lo cual coincide con lo expresado por los estudiantes (MINERD, 2018, pp. 30-31). Tres participantes lo expresan como sigue:

a) Desde el inicio hasta el final de la clase, me sentí motivada y feliz de conocer nuevas estrategias y ver el progreso que he obtenido.

b) Al inicio, puede llegar a ser abrumador ver todos los desafíos por delante, pero en el desarrollo nos dimos cuenta de toda la capacidad sin explotar que teníamos. c) Al principio estaba un poco asustado, porque me habían dicho que el docente ponía muchas asignaciones y uno tendría que trabajar bastante. Sin embargo, aunque fueron bastantes las tareas y lo que uno tuvo que hacer para el blog fue mucho, debo decir que terminé bien contento y siento que aprendí bastante.

En el primer comentario, la joven vincula "motivada" con "progreso". En el segundo, el estudiante conecta "abrumador" con "desafío" y con "capacidad sin explotar". En el tercero, aparecen en el mismo plano términos como "asustado", "contento" y "siento que aprendí bastante". Como se puede inferir, el hilo conductor resultante tanto del aula invertida y, en especial, del TPACK, ha sido un impacto emocional positivo debido a que asumir el protagonismo de su aprendizaje les parece retante, en un inicio, pero dado el uso de herramientas digitales pertinentes, el proceso y el resultado son reconfortantes.

En ese aspecto, los hallazgos coinciden con otros, de estudios realizados en Chile, en profesores de Física: "Durante el curso los estudiantes pudieron aprender los contenidos de manera activa" (Hernández y Tecpan, 2017). De igual modo: "En conclusión, mediante la implementación del modelo TPACK, en el micro currículo de Matemática, se puede establecer que las metodologías de enseñanza favorecen un aprendizaje más eficiente y eficaz; además, es innovador, participativo e interactivo" (Mejía, 2020, p. 80). En una palabra, el impacto del TPACK en el estudiantado deviene en un empoderamiento.

En lo referente a integrar la tecnología al proceso de enseñanza-aprendizaje para eficientizar el desarrollo de las competencias, cabe destacar que los recursos tecnológicos favorecen la implementación del aula invertida y, como resultará obvio, del TPACK. Esto a sabiendas de que se mantiene el temor de que la tecnología sea un distractor del proceso de enseñanza-aprendizaje: "Los riesgos o desventajas también aparecen, desafortunadamente, como alcanzar niveles altos de adicción generando mayores distracciones, pérdidas de tiempo...". (Quiroga et al., 2019, p. 78). Otros estudios plantean dudas similares: "Pero también puede significar algunas desventajas, como el hecho de que los (...) alumnos no tomen tan en serio las actividades 
académicas, dada la facilidad con la que obtienen la información" (Gómez et al., 2016, p. 63). Por otra parte, se manifiesta lo siguiente: "El 17\% de los jóvenes afirma que utiliza las TIC para distraerse en clase, resultando significativamente alta la cantidad de alumnos de PCPI que se distrae mucho en clase" (Díaz et al., 2019, p. 5). Por último, se enfatiza que la libre interacción de los estudiantes los recursos puede llevarlos a confundir el conocimiento con la información o el cúmulo de datos (Paucar, 2019, p. 25).

En el presente estudio los resultados apuntan en la dirección opuesta a los planteamientos anteriores, ya que las TAC (usadas conscientemente y con la guía del docente) posibilitan el aprendizaje significativo y autónomo, así como el desarrollo de competencias. De hecho, el uso de las TAC favorece la evolución de cada estudiante, en relación con lo que Vygotsky denominó la Zona de Desarrollo Próximo: “El papel del más experto consiste en aportar (organizar de cierta manera) los elementos que constituyen la ayuda o guía para promover el conocimiento del mundo y el desarrollo, habida cuenta de que ambos acontecen en el curso del enfrentamiento y la solución del problema" (Labarrere, 2016, p.47).

Uno de los estudiantes lo valoró como sigue: "La metodología fue muy dinámica y creativa, ajustándose a las competencias de la asignatura y la manera de dirigir la clase fue muy pedagógica. Los recursos muy innovadores y oportunos".

En el mismo tenor, otra estudiante resalta la relevancia de la integración de la tecnología:

En lo personal, me encantó lo impartido a lo largo de la asignatura, todo el contenido tratado era interesante y de buena utilidad para nuestra futura profesión, lo que más me emociona de lo que he aprendido durante la asignatura es que tengo más herramientas para poder impartir clases de forma dinámica y soy más capaz de realizar actividades que generen un aprendizaje significativo en mis estudiantes.

Las aseveraciones anteriores denotan que esta alumna valoró el desarrollo de las clases como interesante y útil, tanto como estudiante como futura docente; no obstante, el empoderamiento que dice experimentar lo vincula a las herramientas con las que trabaja. En este punto, es preciso destacar que, al inicio, cuando se les pregunta sobre si utilizan o no los recursos principales que se abordan en la asignatura, la mayoría contesta indicando que, en sus prácticas, trabajan con el aprendizaje basado en problemas y con Kahoot; sin embargo, no señalan el uso del blog, ni de los demás recursos digitales; tampoco se refieren al aula invertida ni al TPACK y no citan recursos tecnológicos distintos al mencionado.

Validando lo anterior, es muy relevante destacar la importancia de utilizar una variedad de recursos, puesto que muchas personas tienden a utilizar el video tutorial, casi de manera exclusiva, para trabajar los contenidos conceptuales (antes de la clase): "En este caso, se realizaron 65 videos con estas características, los cuales se pusieron a disposición de los estudiantes en la plataforma institucional de la Universidad" (Domínguez, et al., 2015, p. 519).

En relación con la implementación de estas metodologías, estrategias y recursos, en su práctica docente, uno de los estudiantes manifiesta:

Profesor, al inicio no me gustó la metodología porque me consumía mucho tiempo y yo sentía que casi toda la responsabilidad del proceso era mía. Tampoco entendía cómo era posible hacer la tarea antes de la clase y no después. También, me sentí un poco abrumado porque eran muchas herramientas digitales distintas. Sin embargo, junto con esta asignatura, estaba cursando Práctica Docente y cuando implementé con mis estudiantes lo que había aprendido en Innovación, el resultado fue maravilloso: la mayoría de ellos se sintió motivado y evidenció un empoderamiento de su aprendizaje. Ellos estaban felices por como estaban aprendiendo y yo me sentía el doble de feliz porque experimenté dos crecimientos, de manera simultánea: como estudiante y como docente.

En síntesis, la aplicación del aula invertida en conjunción con el TPACK y con las TAC deriva en los siguientes beneficios para los estudiantes: se propician el aprendizaje significativo, autónomo y colaborativo, así como el desarrollo de las competencias, del pensamiento lógico, creativo y crítico, la capacidad para la autorregulación y el uso provechoso del tiempo, la automotivación y el 
empoderamiento. En cuanto al rol de los docentes, estos pueden convertirse en guías y facilitadores, así como orientar su docencia al desarrollo de las competencias de sus estudiantes. Además, se propicia el uso de las herramientas tecnológicas pertinentes, así como el fomento de la capacidad crítica y creativa de los estudiantes. De este modo, sin descuidar el resultado, se enfatiza el proceso.

Por último, es preciso destacar que es importante considerar el contexto en el que ocurre el proceso de enseñanza-aprendizaje. En este sentido, las edades, los niveles, el acceso a los recursos, los perfiles de ingreso y egreso, las circunstancias, el nivel de dificultad, el tiempo implicado y la realidad de cada grupo debe ser tomada en cuenta. Un dato interesante es que, a pesar de que los estudiantes tienden a concebirse como nativos digitales, en muchos casos requieren la guía del docente en el aspecto tecnológico. Por otra parte, tampoco se puede generalizar y afirmar que el aula invertida funcionará siempre y en todos los contextos, así como tampoco se puede aseverar que con tutoriales el estudiante puede lograr total y exitosamente la autonomía de su aprendizaje o el dominio de las TAC. No obstante, sí se comprobó un mayor nivel de compromiso, entusiasmo, aprendizaje, autonomía y empoderamiento de los estudiantes.

\section{Referencias bibliográficas}

Aguilera, C., Manzano, A., Martínez, I., Lozano, M. y Casiano, C. (2017). La psicología hoy; retos, logros y perspectivas de futuro. INFAD, 4(1), 261-263. https://doi.org/10.17060/ijodaep.2017.n1.v4.1055

Ausubel, D. (2002). Adquisición y retención del conocimiento. Paidós.

Bergmann, J. y Sams, A. (2014). Dale vuelta a tu clase. Ediciones SM.

Constantino, I. y Castillejos, A. (2020). El modelo tpack en la práctica B-learning de los docentes de Matemáticas. Memoria del Congreso de Docencia, Investigación e Innovación Educativa, (págs. 468-488). https://r.issu.edu.do/l?|=105122mS

Díaz, A., Mercader, C. y Gairín, J. (2019). Uso problemático de las TIC en adolescentes. REDIE, 21(e07), 1-11. https://doi.org/10.24320/ redie.2019.21.e07.1882

Domínguez, L., Vega, N., Espitia, E., Sanabria, Á., Corso, C., Serna, A. y Osorio, C. (2015). Impacto de la estrategia de aula invertida en el ambiente de aprendizaje en cirugía. Biomédica, 35(4), 513-521. http://dx.doi. org/10.7705/biomedica.v35i4.2640

García, M., Reyes, J. y Godínez, G. (2017). Las TIC en la educación superior, innovaciones y retos. Revista Iberoamericana de Ciencias Sociales y Humanísticas, 6(12), 299-316. https://doi.org/10.23913/ricsh.v6i12.135

Gómez, I. (2015). El modelo TPACK en los estudios de grado para la formación inicial del profesorado en TIC. Didáctica Geográfica, 16, 185-201. https://doi. org/10.15366/tp2016.28.010

Gómez, M., Contreras, L. y Gutiérrez, D. (2016). El impacto de las tecnologías de la información y la comunicación en estudiantes de Ciencias Sociales. Innovación Educativa, 16(71), 6180. https://r.issu.edu.do/l?|=10515hph

González, M., Ojeda, M. y Pinos, P. (2020). Desafío del Siglo XXI en la educación: dando saltos del TIC-TAC al TEP. Revista Scientific, 5(18), 323-344. https://doi.org/10.29394/Scientific. issn.2542-2987.2020.5.18.17.323-344

Goodwin, B. y Miller, K. (2013). Evidence on Flipped Classrooms Is Still Coming In. Educational Leadership, 70(6), 78-80. https://r.issu. edu.do/l?|=10518Pbs

Hamdam, N., McKnight, P., McKnight, K. y Arfstrom, K. (2013). Review of Flipped Learning. https://r.issu.edu.do/l?l=10517Auh

Hernández, C. y Tecpan, S. (2017). Aula invertida mediada por el uso de plataformas virtuales. Estudios Pedagógicos, 43(3), 193-204. http://dx.doi.org/10.4067/ S0718-07052017000300011 
Instituto Tecnológico de Monterrey. (2014). Aprendizaje invertido. México: Observatorio de Innovación Educativa. https://observatorio. tec.mx/edutrendsaprendizajeinvertido

King, A. (1993). From sage on the stage to guide on the side. https://doi.org/10.1080/8756755 $\underline{5.1993 .9926781}$

Koehler, M., Mishra, P. y Cain, W. (2015). ¿Qué son los saberes tecnológicos y pedagógicos del contenido (TPACK)? virtualidad, educación y ciencia, 10(6), 9-23. https:// www.punyamishra.com/wp-content/uploads/2016/08/11552-30402-1-SM.pdf

Labarrere, A. (2016). Zona de Desarrollo Próximo como eje del desarrollo de los estudiantes: de la ayuda a la colaboración. Summa Psicológica, 13(1), 45-56. https://doi. org/10.18774/448x.2016.13.293

Latorre, A. (2005). La investigación-acción. Grao.

Latorre, E., Castro, K. y Potes, I. (2018). Las TIC, las TAC Y las TEP: innovación educativa en la era conceptual. Universidad Sergio Arboleda.

L'Ecuyer, C. (2018). ¿Son nuestros alumnos nativos digitales? EDUforics. https://r.issu. edu.do/l?|=10519tgR

Marín, D., Santana, P. y Castro, M. (2021). Escuela digital: estrategias y materiales didácticos digitales en Educación Infantil y Primaria. Revista Iberoamericana de Educación, 85(1), 9-13. https://doi. org/10.35362/rie8514179

Mejía, D. (2020). Implementación del modelo TPACK en el plan micro-curricular de matemática dirigida a los estudiantes del primer año de bachillerato general unificado de la Institución Educativa Fiscal Amazonas en el periodo 2018- 2019. Tesis de grado, Universidad Central del Ecuador, Facultad de Filosofía, Letras y Ciencias de la Educación. https://r.issu.edu.do/l?|=10513uuN
Ministerio de Educación de la República Dominicana. (2018). Diseño Curricular. Santo Domingo.

Paucar, Y. (2019). Aplicación de las TIC en la Educación Peruana. Tesis de la Universidad Nacional de Tumbes, Facultad de Ciencias Sociales. https://r.issu.edu.do/l?|=10516gDP

Posada, F. (08 de 05 de 2013). Canaltic.com. https://canaltic.com/blog/?p=1677

Quiroga, L., Vanegas, O. y Pardo, S. (2019). Ventajas y desventas de las TIC en la educación. Revista de Educación y Pensamiento, 77-85. https://r.issu.edu.do/ |?|=105147mj

Salas, R. (2021). Modelo tpack: ¿Medio para innovar el proceso educativo considerando la ciencia de datos y el aprendizaje automático? Entre Ciencias, 7(19), 51-66. http://dx.doi.org/10.22201/ enesl.20078064e.2018.19.67511

Tobón, S. (2006). Aspectos básicos de la formación basada en competencias. ResearchGate.net. https://r.issu.edu.do/l?|=104461WF

Torres, C., Espinosa, W., Romero, D., Herrera, R. y Herrera, D. (2021). TPACK: Aplicabilidad docente del modelo en Educación General Básica Elemental. Espacios, 42(3), https://r. issu.edu.do/l?!=10650lXg

Villa, A. y Poblete, M. (2007). Aprendizaje basado en competencias. Universidad de Deusto 